\title{
Reflexiones didácticas sobre algunos razonamientos lógicos con la primera ley de Newton y su relación con las ideas previas de los alumnos
}

Didactic reflections on some logical reasonings with the Newton's first law and his relation with the previous ideas of the pupils

\author{
Arturo Carcavilla*1@ , María Lucía Puey² \\ ${ }^{1}$ IES Ramón y Cajal de Huesca, Spain \\ ${ }^{2}$ Universidad de Zaragoza Facultad de Ciencias, Zaragoza, Spain
}

Recibida en 3 de Octubre, 2018. Revisado en 3 de Diciembre, 2018. Aceptado en 6 de Diciembre, 2018.

\begin{abstract}
Las ideas previas de los alumnos que consideran la fuerza como causante de la velocidad de los cuerpos están muy extendidas y son muy resistentes al cambio. Reflexionamos sobre algunos aspectos de los contenidos correspondientes a la primera ley de Newton, que aparece en libros de texto de enseñanza secundaria, ya que pueden resultar equívocos, si el profesor no tiene en cuenta algunas cuestiones lógicas, que, por otra parte redundarían en una mejor formación en pautas de pensamiento, concepciones epistemológicas y conocimiento del método científico de los alumnos. Analizamos algunos libros de texto de enseñanza secundaria, en estos aspectos lógicos en los que el profesor debe de estar atento. Mostramos la conveniencia de introducir pronto una definición de la fuerza newtoniana y comprobar su utilidad en el desarrollo del tema.

Palabras clave: Razonamiento con el condicional, Primera ley de Newton, Errores conceptuales sobre fuerzas, Psicología del pensamiento.
\end{abstract}

\begin{abstract}
Students' previous assumptions that consider the force as the cause of the bodies' speed are widespread and very resistant to change. We reflect on some aspects about the contents of Newton's first law which appear in textbooks of secondary education, since they can be misleading if the teacher does not keep in mind some logical questions that, on the other hand, can lead to a better education in patterns of thinking, epistemological conceptions and students' knowledge of the scientific method. We analyze these logical aspects, where the teacher must pay more attention, in some secondary education textbooks. We show the convenience of introducing soon a definition of Newtonian force and prove its utility in the topic development.

Keywords: Reasoning with conditional, Newton's First law, Conceptual mistakes about forces, Psychology of thinking.
\end{abstract}

\section{Introducción}

En la etapa correspondiente a la enseñanza secundaria, uno de los objetivos que se pretende conseguir es que los alumnos conozcan y comprendan cuestiones fundamentales de ciencia: conceptos, leyes, teorías, etc., en particular de física, y es también muy importante que adquieran conocimientos básicos sobre el método científico, un saber hacer (estrategias de conocimiento) y unas concepciones epistemológicas adecuadas.

Han sido muy estudiadas las ideas previas [1] sobre conceptos como la fuerza, el movimiento, la luz, el calor, la temperatura, etc. Del mismo modo, se han estudiado [2] las pautas de pensamiento, las concepciones epistemológicas y otros elementos que, en cierto modo podríamos denominar preconcepciones sobre los aspectos básicos de

*Correo electrónico: arcarcas@ono.com lo que es la ciencia, el método científico, la comprensión, la explicación, el aprendizaje y otras cuestiones similares. Nos proponemos los siguientes objetivos:

1. Mejorar la comprensión del concepto de fuerza y su relación con la velocidad. Por una parte debemos mostrar claramente la definición de fuerza, cosa que suele hacerse, pero no pretender que se deriva de la primera ley de Newton. Por otra, hemos de justificar el por qué eliminar en nuestra definición las "fuerzas"mantenedoras de la velocidad. El argumento principal, de tipo cuantitativo, está relacionado con la segunda ley de Newton, que se cumple utilizando las fuerzas "newtonianas". Uno de los argumentos, de tipo cualitativo que podrían utilizase explícitamente se basa en el precepto metodológico conocido como la navaja de Ockam, o una referencia epistemológica similar, adaptada a la terminología actual, [3] como indicamos en el 
apartado 4. Además, de este modo, mostraríamos un ejemplo más de que en la ciencia, los conceptos deben ser precisamente definidos para no conducir a inconsistencias [4].

2. Mostrar razonamientos relacionados con la primera ley de Newton, utilizando ejemplos extraídos de algunos libros de texto de Física y Química. Algunos consisten en hacer deducciones que no pueden hacerse lógicamente: obtener una proposición recíproca a partir de una condicional directa, por ejemplo, a partir de la proposición "si un cuerpo está acelerado, actúa una fuerza sobre él" a $\rightarrow \mathrm{f}$ (primera ley de Newton), pretender que puede deducirse la proposición: "si actúa una fuerza sobre un cuerpo, éste está acelerado"f $\rightarrow$ a (de la definición de Newton de lo que es fuerza). Esto ocurre a veces de este modo directo, pero, más habitualmente, de modo indirecto, como veremos en el apartado 5 .

3. Mediante una utilización explícita de los razonamientos correctos se puede poner a los alumnos en la dirección adecuada para mejorar algunas de sus pautas de pensamiento, concepciones epistemológicas y conocimiento del método científico. Volveremos a estas cuestiones en los apartados 2 y 4.

4. Por lo que respecta a las pautas de pensamiento y razonamiento de los alumnos, conviene mostrar en lo posible un razonamiento formal para ir acostumbrándolos y para ir tratando de eliminar sus metodologías superficiales, [4]. Además destacaríamos la coherencia del conocimiento científico y su organización lógica explícita, que lo diferencia del conocimiento de la vida cotidiana [4]. En cualquier caso, debemos buscar la transposición didáctica adecuada [5], huyendo de formalismos lógicos extremos pero poniendo de manifiesto las relaciones lógicas que existan. Por ejemplo, a partir de la proposición "si un cuerpo está acelerado, actúa una fuerza sobre él" a $\rightarrow$ f, no podemos descartar por motivos lógicos que no esté acelerado y que actúe una fuerza sobre él, esto es, la existencia de fuerzas mantenedoras de la velocidad. Si aceptamos una proposición condicional, cuando el antecedente es falso, el consecuente puede ser verdadero o falso. Esto debe aclararse explícitamente utilizando la definición de fuerza de tipo newtoniano, pero si no lo hacemos, no podemos evitar que los alumnos mantengan como posible la existencia de estas fuerzas mantenedoras de la velocidad que, además son parte importante de sus ideas previas. Por lo que respecta a las concepciones epistemológicas, destacamos los principios generales, frente a resultados específicos [2]. Al mejorar la estructura lógica, como también pretendemos, orientaríamos a los alumnos a considerar la estructura del conocimiento científico como un todo coherente, frente a la consideración que tienen muchos alumnos, que lo consideran organizado en piezas sueltas [6].

5. No pretendemos entrar en la metodología que pueda usar el profesor para conseguir que sus alumnos alcancen el aprendizaje deseado. Aceptando que deben construir un aprendizaje significativo y que deben resolverse los conflictos cognitivos que les surjan, consideramos que el profesor debe elegir si prefiere una enseñanza más o menos expositiva o del tipo redescubrimiento orientado u otras. Por otra parte, como indicamos en el apartado 7.i, actualmente coexisten tres teorías que tratan de dar cuenta del comportamiento de la mente humana al resolver problemas de razonamiento. No nos posicionamos a este respecto y únicamente señalamos posibles defectos en el razonamiento lógico, como uno de tantos factores que influyen en el aprendizaje.

\section{Ideas previas, pautas de pensamiento y concepciones epistemológicas de los alumnos}

Es bien conocida desde el trabajo de Viennot [7], la importancia de las ideas previas de los alumnos para el aprendizaje de la ciencia. Estas ideas son científicamente incorrectas, inconexas y muy resistentes al cambio. El concepto de fuerza y su papel en las leyes del movimiento son de los más problemáticos y han sido muy estudiados. El punto que tiene una relación más estrecha con las cuestiones que vamos a analizar es el de la relación entre fuerza y velocidad, $[7,8]$. El aspecto más importante de esta relación es creer que si existe una velocidad o componente de la velocidad en una dirección dada, entonces existe una fuerza en esa dirección. Podemos encontrar muchas referencias en [1]. Vamos a ver ejemplos de tres trabajos que estudian estas ideas.

1. En un estudio hecho con escolares londinenses, utilizando el método de la entrevista sobre ejemplos, Watts [8] averiguó los diferentes marcos alternativos que los alumnos entrevistados tenían sobre el concepto de fuerza. Clasifica los resultados en ocho marcos, a los que da diferentes nombres, como fuerzas sustanciales (similares a la noción física de presión), operativas (similares a la noción de energía), motoras (causa del mantenimiento del movimiento), de impacto (similares al concepto de momento), y así hasta ocho. El marco conceptual que nos interesa en nuestro análisis es el de las fuerzas que llama motoras, que los alumnos conciben como necesarias para mantener la velocidad.

2. Ha sido importante el estudio pionero de Viennot [7], uno de cuyos test fue el siguiente:

Dibuja las fuerzas que actúan sobre la pelota, lanzada por el muchacho de la figura 1: a) Cuando está subiendo, b) cuando está en el punto más alto 


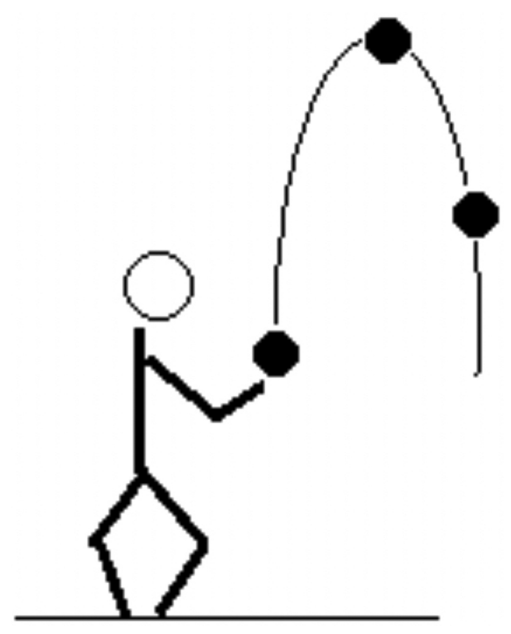

Figura 1: Muchacho lanzando una pelota

de la trayectoria, c) cuando está descendiendo.

Porcentaje de respuestas erróneas:

Estudiantes franceses de último año de secundaria: $61 \%$

Estudiantes franceses de primer año de universidad: $42 \%$

Estudiantes belgas de primer año de universidad: $56 \%$

3. Por último mostramos otro estudio, también similar hecho en España por Carrascosa y Gil (1982) [9]

Decir si son verdaderas o falsas las siguientes afirmaciones:

a) Si sobre un cuerpo no actúa ninguna fuerza o la fuerza resultante es nula, deberá estar en reposo

b) El movimiento de un cuerpo siempre tiene lugar en la dirección de la fuerza resultante.

c) Si en un instante la velocidad de un cuerpo es nula, la fuerza resultante en ese mismo instante también lo será.

Los resultados, sobre el concepto de fuerza son los siguientes:

$\begin{array}{lccc}\text { Curso } & \mathrm{N}^{\circ} & \text { Todas mal } & \begin{array}{c}\text { Alguna o } \\ \text { todas mal }\end{array} \\ 2^{\circ} \mathrm{BUP} & 196 & 49,5 & 100 \\ 3^{\circ} \mathrm{BUP} & 213 & 52,1 & 99,5 \\ \mathrm{COU} & 181 & 50,3 & 97,8 \\ \text { Magisterio } & 145 & 60.0 & 100 \\ 2^{\circ} \text { Química } & 140 & 53,0 & 95,1\end{array}$

Los resultados se comentan por sí mismos.

Estos y otros muchos estudios ponen de manifiesto la persistencia de las ideas previas a pesar de la instrucción recibida, particularmente la relación entre fuerza y velocidad. Se constata que para un gran número de alumnos, la fuerza está asociada a la velocidad. Algo similar al impetus de Buridán [10]

Otro tema importante es el de las pautas de pensamiento y razonamiento de los alumnos [2]. Los alumnos recurren con frecuencia a metodologías superficiales y también pueden aplicar pautas de razonamiento poco científicas en tareas propias de ciencias, en particular heurísticas, como tanteo, reglas empíricas, etc. frente a razonamiento formal. Por lo que respecta a las concepciones epistemológicas [2], en general la física se concibe como una colección de fórmulas y símbolos que se refieren a los conceptos a los que articulan y también está más orientada a resultados específicos que a principios generales.

Hay una serie de diferencias entre el conocimiento científico y el conocimiento de la vida cotidiana en lo que respecta a finalidades, requerimientos, validez, comprensión, especificación de conceptos, organización del conocimiento, métodos, control de calidad y eficiencia [4]. Nos interesan aquí especialmente las diferencias en la organización del conocimiento. Las más interesantes para las cuestiones que vamos a tratar son las siguientes: el conocimiento científico es altamente coherente y está organizado lógicamente de forma explícita, mientras que en la vida cotidiana, el nuevo conocimiento puede ser sólo parcialmente coherente y es almacenado junto con el contexto de aprendizaje pero sin integración global. Por lo que se refiere a la especificación de conceptos, indican que en la vida cotidiana hay poca precisión en la relación conceptos-referentes mientras que en la ciencia deben ser precisamente definidos para no conducir a inconsistencias.

Las concepciones epistemológicas influyen en las estrategias de aprendizaje. Se consideran tres dimensiones [2]: estructura del conocimiento científico (organizado en piezas sueltas o formando un todo coherente), concepciones sobre el contenido físico (fórmulas frente a conceptos) y concepciones sobre el aprendizaje de la física (proceso de construcción o proceso de recepción). En el caso que vamos a tratar, una estructuración lógica adecuada contribuirá a ver el conocimiento científico formando un todo coherente y también resaltaría la importancia de los conceptos. Para ello es importante el tratamiento adecuado del concepto de fuerza.

\section{Cuestiones lógicas elementales. La condicional lógica}

Como acabamos de indicar, el conocimiento científico está organizado lógicamente de forma explícita. Vamos a señalar muy brevemente algunas cuestiones de lógica elemental, fundamentales en este trabajo.

De acuerdo con Quine, la lógica es el estudio sistemático de las verdades lógicas y una oración es lógicamente verdadera si lo son todas las que tienen la misma estructura gramatical [11]. Los hechos lógicos se representan mediante fórmulas cuya interpretación está libre de la 
confusión que tiñe tan fácilmente la expresión lingüística. [12] Se consideran tres partes en el estudio de la lógica teórica que son: el cálculo de proposiciones, el cálculo de clases y el cálculo de predicados. Se denomina proposición a toda oración de la cual tenga sentido afirmar que su contenido es verdadero o falso. Por ejemplo, "la nieve es blanca". En el cálculo de proposiciones no se entra en la estructura más fina, que se manifiesta en la relación entre el sujeto y el predicado, sino que se consideran como un todo, en su conexión lógica con otras proposiciones.

Las proposiciones se pueden conectar lógicamente con otras proposiciones mediante las palabras "y", "o", "noz "si...entonces". Se define la verdad de la proposición compuesta en función de la verdad de las proposiciones constituyentes, de un modo que puede representarse por las tablas de verdad.

Las tablas de verdad de las condicionales directa "si a entonces f", recíproca "si f entonces a", contraria "si no a entonces no fz contrarrecíproca "si no f entonces no a"las mostramos en la tabla 1 (designamos la negación con $\left(^{\prime \prime}\right)$ :

Notemos que la directa y la contrarrecíproca son equivalentes, así como la recíproca y la contraria.

En el condicional directo $\mathrm{a} \rightarrow \mathrm{f}$, cuando el antecedente es falso, puede ocurrir cualquiera de las dos opciones, esto es, que el consecuente sea falso o que el consecuente sea verdadero.

\section{Enunciados de la primera ley de newton y de la definición de fuerza. algunas consecuencias de los mismos}

La forma en que se enuncia habitualmente la primera ley de Newton es la siguiente:

"Todo cuerpo permanece en estado de reposo o mo-
vimiento rectilíneo uniforme a menos que haya una
fuerza que actúe sobre él".
Dicho de otro modo, "Todo cuerpo no está acelerado
a menos que haya una fuerza que actúe sobre él".
Simbólicamente puede representarse a $\rightarrow \mathrm{f}$

"Todo cuerpo permanece en estado de reposo o movimiento rectilineo uniforme a menos que haya una fuerza que actúe sobre él". Simbólicamente puede representarse $\mathrm{a} \rightarrow \mathrm{f}$
Este enunciado es directamente asimilable a una proposición del tipo $4_{1}$ de la tabla 2 . Mostramos en dicha tabla seis enunciados de la primera ley de Newton, equivalentes, utilizando unas expresiones simplificadas. Utilizaremos el siguiente significado de las proposiciones $\mathrm{f}, \mathrm{f}^{\prime}$, a y a'. La proposición f significa que sobre un cuerpo actúa una fuerza f (se entiende que es la fuerza total); la proposición $\mathrm{f}^{\prime}$ significa que no actúa ninguna fuerza o que la fuerza total que actúa sobre el cuerpo es cero; la proposición a significa que el cuerpo está moviéndose aceleradamente; la proposición a significa que el cuerpo no está acelerado, por tanto está en reposo o se mueve con movimiento rectilíneo uniforme.

En este caso la proposición recíproca también es cierta y por ello todos los enunciados recíprocos también son ciertos, lo que nos puede producir alguna confusión. Como ayuda para asegurarnos de distinguir los enunciados directos y recíprocos, podemos utilizar una proposición como la siguiente: si llueve, la calle se moja, que además de ser evidente, tiene una proposición recíproca que es falsa.

Llover $(\mathrm{Ll}) \rightarrow$ mojarse la calle $(\mathrm{M})$ pero no es cierto que mojarse la calle $\rightarrow$ llover.

De un modo más simplificado podemos decir que $\mathrm{Ll} \rightarrow$ $\mathrm{M}$ es cierto (mostramos todos los enunciados en la tabla $3)$, pero $\mathrm{M} \rightarrow \mathrm{Ll}$ no lo es.

Si llueve se debe mojar la calle pero sabemos que es posible que no llueva y se moje la calle. Hay modos de mojar la calle distintos de la lluvia. En el caso de la primera ley de Newton, si el cuerpo está acelerado, deberá actuar una fuerza pero, de acuerdo con el enunciado y

Tabla 3: Diferentes formulaciones condicionales, todas ellas equivalentes, de un caso evidente

\begin{tabular}{l}
\hline $1_{1}$ - Si llueve, la calle se moja \\
\hline $2_{1}$ - Si no se moja la calle, no llueve \\
\hline $3_{1}$ - Sólo si se moja la calle, llueve \\
\hline $4_{1}$ - No llueve, a menos que se moje la calle \\
\hline $5_{1}$ - Es suficiente que llueva para que la calle se moje \\
\hline $61_{1}$ - Es necesario que se moje la calle para que llueva \\
\hline
\end{tabular}

Tabla 1: Tablas de verdad de proposiciones, objeto de nuestro estudio

\begin{tabular}{|c|c|c|c|c|c|}
\hline & & Directa $\left(1_{1}\right)$ & Recíproca $\left(1_{2}\right)$ & Contraria $\left(2_{2}\right)$ & Contrarrecíproca $\left(2_{1}\right)$ \\
\hline $\mathrm{a}$ & $\mathrm{f}$ & $a \rightarrow f$ & $\mathrm{f} \rightarrow \mathrm{a}$ & $a^{\prime} \rightarrow f^{\prime}$ & $f^{\prime} \rightarrow a^{\prime}$ \\
\hline $\mathrm{V}$ & $\mathrm{V}$ & $\mathrm{V}$ & $\mathrm{V}$ & $\mathrm{V}$ & $\mathrm{V}$ \\
\hline $\mathrm{V}$ & $\mathrm{F}$ & $\mathrm{F}$ & $\mathrm{V}$ & $\mathrm{V}$ & $\mathrm{F}$ \\
\hline $\mathrm{F}$ & $\mathrm{V}$ & $\mathrm{V}$ & $\mathrm{F}$ & $\mathrm{F}$ & $\mathrm{V}$ \\
\hline $\mathrm{F}$ & $\mathrm{F}$ & $\mathrm{V}$ & $\mathrm{V}$ & $\mathrm{V}$ & $\mathrm{V}$ \\
\hline
\end{tabular}

Tabla 2: Diferentes formulaciones condicionales de la primera ley de Newton, todas ellas equivalentes $1_{1}$ - Si un cuerpo está acelerado (si a), actúa una fuerza sobre él (entonces f). Se puede simbolizar: $[\mathrm{a} \rightarrow \mathrm{f}]$ $2_{1}$ - Si no actúa una fuerza sobre él (si no f), no está acelerado (no a). Se puede simbolizar: [f' $\rightarrow \mathrm{a}^{\prime}$ $3_{1}$ - Sólo si actúa una fuerza sobre él (sólo si f), está acelerado (entonces a) $[\mathrm{a} \rightarrow \mathrm{f}]$ $4_{1}$ - No está acelerado (no a), a menos que actúe una fuerza sobre él (a menos que f) $[\mathrm{a} \rightarrow \mathrm{f}]$ $5_{1}$ - Es suficiente que esté acelerado (es suficiente a) para que actúe una fuerza sobre él (para que f) $[\mathrm{a} \rightarrow \mathrm{f}]$ $6_{1}$ - Es necesario que actúe una fuerza sobre él (es necesario f) para que esté acelerado (para que a) $[\mathrm{a} \rightarrow \mathrm{f}]$ 
por razones lógicas puramente, es posible que no esté acelerado y que actúe una "fuerza". Podría haber "fuerzas" que no provoquen la aceleración. Recordemos que muchos alumnos consideran la "fuerza" como causante de la velocidad como hemos visto al recordar las ideas previas de los alumnos. Está claro que lo que entonces llamaríamos "fuerzas" sería lo que muchos alumnos consideran que es la fuerza y no lo que Newton y nosotros consideramos que es fuerza.

Como señala Anderson (1990), [13] la definición de fuerza que Newton [14] presenta en sus Principia es una afirmación suficiente del papel de las fuerzas en el cambio de estado mientras que la primera ley es una afirmación necesaria.

Definición de fuerza, según Newton: "La fuerza im-
presa es una acción ejercida sobre un cuerpo para
cambiar su estado, bien sea de reposo o de movimien-
to uniforme en línea recta". Diríamos con nuestro
simbolismo que $\mathrm{f} \rightarrow$ a.

Dicho de otro modo, si una fuerza impresa actúa sobre un cuerpo, cambia su estado de reposo o de movimiento rectilíneo, o sea, le produce aceleración, por eso, podemos representar esta definición de un modo simplificado como $\mathrm{f} \rightarrow$ a. Esta definición elimina las fuerzas asociadas a la velocidad, cosa que no hace la primera ley. Utilizamos aquí la expresión fuerza impresa porque es una cita literal de Newton, pero en modo alguno pretendemos que la utilicen los alumnos.

Mostraremos en el apartado 6 los contenidos de la parte de algunos libros de texto en que se trata de la primera ley de Newton

Básicamente se comienza en todos los casos con el enunciado de la primera ley de Newton y posteriormente se enuncian algunas proposiciones. Vamos a dar unas ideas de carácter general de lo que se hace y luego, en el apartado 6 , describiremos con más detalle cómo se trata en cada libro.

Enunciado de partida:

"Todo cuerpo permanece en estado de reposo o movimiento rectilíneo uniforme a menos que haya una fuerza que actúe sobre él". Dicho de otro modo, un cuerpo no está acelerado a menos que actúe una fuerza sobre él. Esto equivale, como podemos ver en la Tabla 2 a "si un cuerpo está acelerado, actúa una fuerza sobre él. Simbólicamente puede representarse $\mathrm{a} \rightarrow \mathrm{f}$

Partimos pues de a $\rightarrow \mathrm{f}$ La simbolizaremos gráficamente mediante un diagrama de Venn como indica la figura 2. Representamos el conjunto de todos los cuerpos sometidos a fuerzas, mediante un óvalo y una parte de ese conjunto, el círculo negro, la forman los cuerpos acelerados. Fuera del óvalo están los cuerpos no sometidos a fuerzas ni acelerados, Creemos que esta representación gráfica resulta más clara para nuestros propósitos que las tablas de verdad.

Este enunciado es compatible con la existencia de movimientos no acelerados sobre los que actúa una "fuerza", que podríamos ver como algo equivalente al impetus medieval [5], una virtus impressa o cualidad impresa en el cuerpo cuya propiedad es moverlo. También es compatible lógicamente con la existencia de movimientos no acelerados y sin fuerzas. Estos aspectos de la $1^{\text {a Ley de }}$ Newton a $\rightarrow$ f están relacionados directamente con las ideas previas de los alumnos, especialmente la posibilidad de movimientos no acelerados con "fuerzas mantenedoras del movimiento". El movimiento uniforme sin fuerzas no se contempla en las ideas previas de los alumnos que estamos considerando. Es lógicamente compatible pero no existiría para los que creen necesaria una fuerza mantenedora del movimiento.

Hay básicamente dos afirmaciones que algunos textos pretenden que se deducen de este principio:

a) La deducción que se hace con más frecuencia es la negación de la necesidad de una fuerza para que un cuerpo se mueva con movimiento uniforme: no es necesaria una fuerza para que el cuerpo pueda moverse con movimiento uniforme, o sea no es cierto $\left[\mathrm{a}^{\prime} \rightarrow \mathrm{f}\right]$. En la figura 2, que representa la primera ley de Newton, vemos que es posible el momento uniforme con fuerzas y sin fuerzas, por tanto, por motivos lógicos no podemos descartar que sea necesaria una fuerza para mantener un movimiento uniforme. Está claro en la representación gráfica, pero insistimos volviendo al ejemplo "si llueve, entonces la calle se moja". Está claro que no es necesario que la calle se moje cuando no llueve, pero es posible, por ejemplo si se riega.

b) Lo más evidentemente equivocado es la deducción, en algún texto, de una proposición recíproca, partiendo

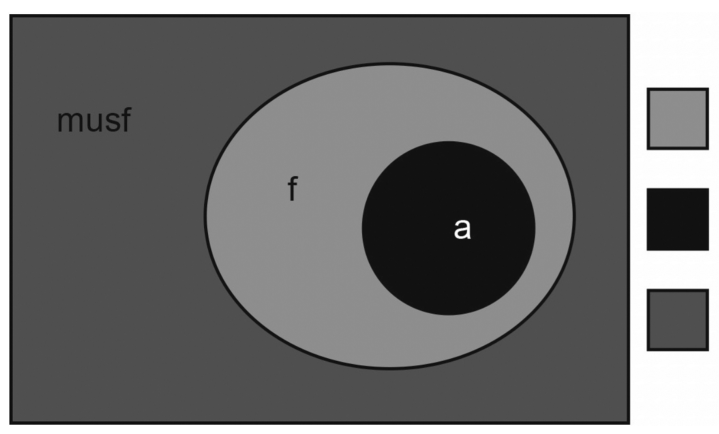

Movimiento no acelerado con fuerzas "mantenedoras del movimiento"

Movimiento acelerado con fuerzas

"newtonianas"

Movimiento uniforme sin fuerzas

Figura 2: 1르 Ley de Newton $a \rightarrow f$ 
de una directa. Evidentemente, un enunciado no se puede justificar apoyándolo en su proposición recíproca. Por ejemplo, diciendo que a $\rightarrow$ f es cierto "porque si existiese una fuerza resultante habría cambios de velocidad" $[\mathrm{f} \rightarrow \mathrm{a}]$. Son proposiciones recíprocas y por tanto independientes. O bien otro texto que dice que si se moviera con velocidad constante, no actuaría ninguna fuerza sobre el cuerpo $\left[\mathrm{a}^{\prime} \rightarrow \mathrm{f}^{\prime}\right]$ que equivale a $[\mathrm{f} \rightarrow \mathrm{a}]$, recíproca de $[\mathrm{a} \rightarrow \mathrm{f}]$.

Recordemos de nuevo la definición de fuerza de Newton:

Definición de fuerza, según Newton: "La fuerza impresa es una acción ejercida sobre un cuerpo para cambiar su estado, bien sea de reposo o de movimiento uniforme en línea recta". Diríamos con nuestro simbolismo que $\mathrm{f} \rightarrow$ a. Podría representarse mediante un diagrama de Venn, como hacemos en la figura 3, de modo similar a la primera ley de Newton.

Esto sí que elimina las fuerzas mantenedoras de movimiento con velocidad constante. Aquello a lo que llamamos fuerza produce aceleración. ¿Por qué esta definición? Porque describe adecuadamente el movimiento, porque con este tipo de fuerzas y la segunda ley de Newton, podemos calcular las aceleraciones de los cuerpos, teniendo las aceleraciones, las matemáticas nos permiten calcular las velocidades y posteriormente, las ecuaciones del movimiento que se ajustan a la realidad. ¿Por qué eliminar en nuestra definición las "fuerzas"mantenedoras de la velocidad? Johsua, y Dupin, [3] en el capítulo en el que describen las referencias epistemológicas de la ciencia, indican, en el apartado "El modelo es pertinente a su objeto", que para producir un modelo teórico, la ciencia debe recortar, en la extremada complejidad de la realidad, aspectos limitados, que pueda saber tratar. Ponen el siguiente ejemplo: tenemos una teoría sobre los circuitos eléctricos, que utiliza determinados conceptos. Supongamos que alguien dice que tal vez el gato que pasa por la calle debería ser incluido en la teoría porque tal vez influya en el comportamiento de los circuitos. Ante una situación así, la respuesta es que quien dice esto proponga una teoría que lo incluya y que describa y prediga adecuadamente, al menos los aspectos de la realidad que describía nuestra teoría. Mientras no lo haga, tenemos el derecho metodológico de ignorarlo. De un modo similar, podemos actuar con las "fuerzas"mantenedoras de la velocidad. Nosotros tenemos una teoría que utiliza las fuerzas newtonianas, que nos ha dado buenos resultados sin utilizar ese otro tipo de fuerzas. Mientras no se nos de otra teoría satisfactoria que las incluya, tenemos el derecho metodológico de ignorarlas [3]. Esta estrategia, ya la encontramos en el siglo XIV en el precepto metodológico conocido como la navaja de Ockham [15], que dice que "no hay que multiplicar los entes sin necesidad".

La primera ley, junto con la definición newtoniana de fuerza dan lugar a la relación entre fuerza y movimiento que admitimos hoy día, según la cual, las fuerzas producen aceleraciones y las aceleraciones son producidas por fuerzas. Lógicamente, la relación entre f y a es la de una equivalencia material y la representamos gráficamente en la figura 4:

Un enfoque de la enseñanza de las leyes de Newton que nos parece adecuado es el del vídeo Física sobre patines, [16], descrito en la siguiente reseña [17].

\section{Resultados del análisis de razonamientos lógicos relacionados con la primera ley de Newton en algunos libros de texto}

Como ya hemos indicado vamos a mostrar los contenidos de la parte de algunos libros de texto que trata de la primera ley de Newton. Estas partes contienen aspectos ante los cuales el profesor debe de estar atento por los motivos que hemos expuesto. El libro de texto es un elemento más del proceso de enseñanza-aprendizaje. En dicho proceso, el profesor es un elemento fundamental que debe utilizar adecuadamente el libro de texto, cosa que siempre podrá hacer, completando aspectos, que falten en su opinión y reconduciendo otros, cuando lo considere oportuno.

Estos libros se utilizaron en España en el curso $2^{\circ}$ de BUP, esto es, $2^{\circ}$ del Bachillerato Unificado Polivalente. Este curso corresponde al actual $4^{\circ}$ de ESO, Educación Secundaria Obligatoria. Los alumnos lo terminaban con dieciséis años si no habían repetido curso. El BUP comenzó en el curso 75-76 y se extinguió definitivamente en el año 2000.

En algunos de los libros que analizamos se da la definición de fuerza newtoniana, antes de hablar de la primera

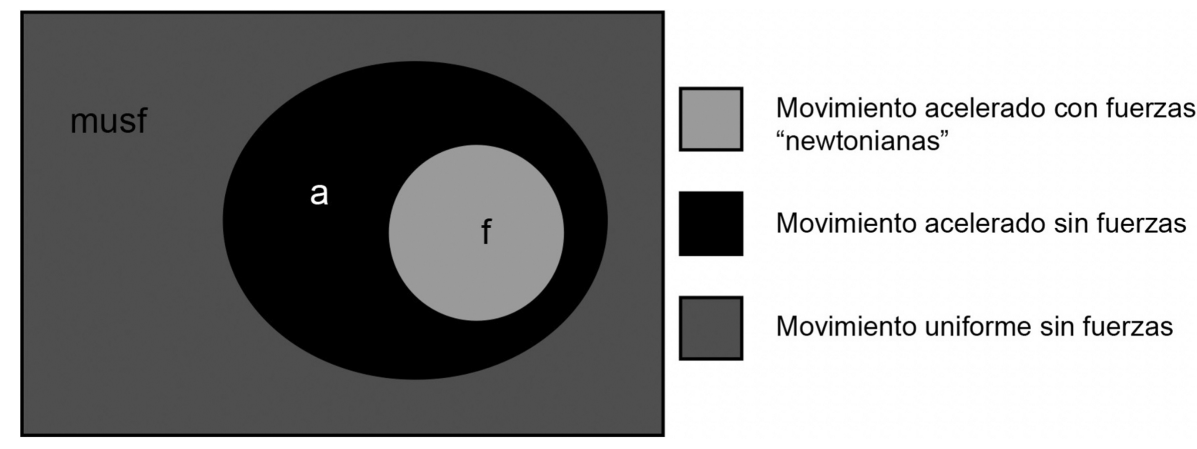

Figura 3: Definición de fuerza newtoniana $\mathrm{f} \rightarrow \mathrm{a}$ 


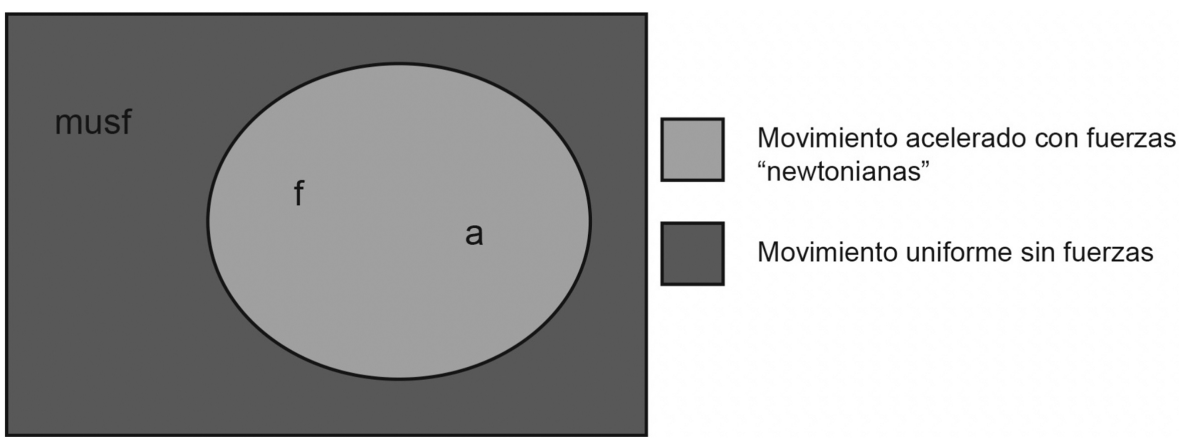

Figura 4: Relación entre fuerza y aceleración, aceptada actualmente por los científicos. a $\leftrightarrow f$

ley de Newton y en otros, después, pero en algunos casos, no se hace alusión a esta definición en el apartado correspondiente a la primera ley y parece que se considera que puede deducirse la no necesidad de una fuerza para mantener un movimiento rectilíneo uniforme sin tener en cuenta el contenido lógico del enunciado de esa ley ni las ideas previas de los alumnos sobre el concepto de fuerza. En cualquier caso, parece que se considera implícitamente que el concepto newtoniano de fuerza es algo evidente. Sin embargo, hemos de tener en cuenta la gran importancia que tienen las ideas previas de los alumnos sobre la fuerza y que es bien sabido que, incluso después de estudiar estas cuestiones, muchos alumnos siguen manteniéndolas. Un razonamiento inadecuado o poco explícito, evidentemente no les va a ayudar a clarificar el concepto en todos sus aspectos. También es muy importante actuar para que las pautas de razonamiento y las concepciones epistemológicas se vayan formando adecuadamente, lo que debe de ser completado por el profesor. Evidentemente, los libros, después de la primera ley de Newton, tratan la segunda y la tercera y esto les da oportunidad de completar el concepto de fuerza y otras cuestiones. Lo único que pretendemos al mostrar estos fragmentos es ilustrar lo que hemos dicho anteriormente, no desaprobar los libros, pues, como ya hemos indicado, los libros son un instrumento más que debe ser utilizado por el profesor.

Transcribiremos primero, para cada libro de texto, el fragmento que deseamos analizar, textualmente, entre comillas y en cursiva. Subrayamos las frases clave. Posteriormente analizamos el fragmento.

Texto 1 del [18]

Comienza revisando aspectos dinámicos de la física del sentido común, mediante tres actividades y luego continúa con lo siguiente:

"Principios fundamentales de la mecánica newtoniana (p. 42, 43)

Concepto cualitativo de fuerza: primer principio.

[Es un diálogo entre Simplicio y Salviati en el cual éste último va preguntando al primero por el movimiento de una bola que se moviera por un plano inclinado, hacia abajo y por otro plano, hacia arriba para concluir preguntándole por lo que pasaría si se dejara en un plano horizontal. Transcribimos esta última parte].
Simplicio: No puedo descubrir ninguna causa de aceleración ni de retardo si no hay inclinaci hacia abajo ni pendiente hacia arriba.

Salviati: Bien, si no hay causa de retardo, menos la habrá para detenerlo, por tanto, ¿qué distancia recorrerá el cuerpo en movimiento?

Simplicio: Pues tanta como la superficie ni inclinada ni ascendente...

Pregunta. A partir de un análisis del diálogo anterior considerar si es cierta o no la siguiente proposición:

Para que un cuerpo permanezca en movimiento ha de estar actuando una fuerza sobre él."

Texto 1 del [18] Análisis

Partimos de que no hay causa de aceleración ni retardo lo que, en una transposición didáctica [7] a nuestros conceptos, podríamos interpretar como que no hay fuerzas de tipo newtoniano, aunque no es estrictamente lo que dice Galileo, que no utiliza la palabra fuerza.

No define el concepto de fuerza. Posiblemente se ha discutido en clase previamente, pero centrándonos en los contenidos presentes, se pretende que el alumno deduzca que no es necesaria una fuerza para que el cuerpo permanezca en movimiento. Si el movimiento es acelerado, sí que es necesaria una fuerza para ese movimiento, es la proposición de partida $\mathrm{a} \rightarrow \mathrm{f}$, pero este no es el problema. La cuestión reside en los movimientos no acelerados. La explicación más clara, que muestra todos los casos compatibles con esta proposición se muestra en la figura 2, donde se ve la posibilidad de que existan movimientos no acelerados sobre los que actúen fuerzas. Recordemos que muchos alumnos consideran las fuerzas como causantes de la velocidad y cuando lanzamos un cuerpo dicen que le hemos dado una fuerza y eso es lo que le permite seguir moviéndose. Esto no es posible si aceptamos la definición newtoniana de fuerza, en cuyo caso las fuerzas son interacciones que únicamente producen aceleraciones. Debemos eliminar las fuerzas productoras de velocidad por innecesarias, como hemos indicado en [3] y [15] y este es un buen momento para hacer una discusión sobre esto. La justificación cuantitativa de nuestra defnición es la segunda ley de Newton, cosa que se puede avanzar de un modo resumido ahora y volver aquí cuando se haya visto esa ley.

Texto 2 del [19]

"Los cambios en el movimiento (p. 60,61) 
Pregunta

¿Estás de acuerdo con esta afirmación?

i) 1. Si un cuerpo se mueve en una dirección es porque alguna fuerza lo está empujando en dicha dirección.

[Posteriormente utiliza el mismo fragmento de diálogo de Galileo que en el libro "La construcción de las ciencias físico-químicas", pero resumido].

Conclusión de la experiencia de Galileo

iii) Todo cuerpo persevera en su estado de reposo o de movimiento rectilíneo uniforme, a no ser que la acción de alguna fuerza le obligue a modificar dicho estado.

Este enunciado corresponde al denominado principio de inercia o primer principio, y fue formulado por Newton.

iv) De acuerdo con el primer principio, no es necesario que actúe una fuerza para que un móvil se mueva en determinada dirección."

Texto 2 del [19] Análisis

Se presenta un enfoque parecido al texto 1 y por tanto, el mismo tipo de problema. El primer principio no define lo que debemos entender por fuerza y según lo que entendamos sería necesario que actúe una fuerza para que el móvil se mueva en una determinada dirección o no lo será. Insistimos en la necesidad de aclarar esto desde todos los puntos de vista, dada la gran importancia de las ideas previas de los alumnos sobre el concepto de fuerza.

Texto 3 del [20]

"Dinámica (p.63)

Principio de la inercia

Cuando un cuerpo está en equilibrio, hemos visto que la resultante de las fuerzas que actúan sobre él es nula y la suma de los elementos de todos los pares de fuerzas es también nula. La experiencia nos dice que tal cuerpo es incapaz de moverse si está en reposo. Tenemos miles de ejemplos en nuestra vida común de cuerpos que permanecen en estado de equilibrio (libros en estanterías, sillas, mesas, etc.)

Cuando un cuerpo que está en equilibrio $(S F=0$, $S M=0)$ está además en movimiento, no tiene por qué detenerse y continuará moviéndose indefinidamente con movimiento rectilíneo y uniforme. Por ejemplo, una bala lanzada por un fusil, de no ser por la atracción de la Tierra (fuerza peso) o por la oposición que presenta el aire a su movimiento, no dejaría de moverse).

Un ejemplo lo tenemos en los cohetes espaciales. Cuando la tercera fase del "Apolo"lanza el módulo de mando...

Por tanto este principio podría enunciarse diciendo:

Todo cuerpo tiende a permanecer en estado de reposo o de movimiento rectilíneo uniforme si existe equilibrio de fuerzas y pares de fuerzas.

Ya que si existiese una fuerza resultante habría cambios de velocidad, y si existiese un par, produciría una rotación."

Texto 3 del [20] Análisis

Ha introducido el concepto de fuerza previamente (no lo mostramos aquí)

Implícitamente elimina las "fuerzas" asociadas al movimiento cuando dice que se puede dar simultáneamente que el cuerpo esté en movimiento y que la suma de fuerzas sea cero. Sin embargo, en el trabajo de clase, debe utilizarse de modo explícito la definición de fuerza y los argumentos mostrados en [3] y [15] que hemos mencionado.

Enuncia el primer principio de este modo: $f{ }^{\prime} \rightarrow \mathrm{a}^{\prime}$ que equivale a $\mathrm{a} \rightarrow \mathrm{f}$ y da una justificación al final que es incorrecta como justificación, ya que es la proposición recíproca: $\mathrm{f} \rightarrow \mathrm{a}$

Texto 4 del [21]

"Dinámica (p. 61)

Principio de Inercia

Una bola que se deja en reposo sobre una mesa permanece inmóvil indefinidamente, mientras no actúe sobre ella alguna fuerza. También estamos familiarizados con la idea de que para que un cuerpo se mueva hay que aplicarle una fuerza. Para arrastrar un armario, sacándolo de su estado de reposo se ha de ejercer una fuerza y cuando se deja de aplicarla el armario se para, debido a la existencia de la fuerza de rozamiento, que se manifiesta entre las superficies de los cuerpos en contacto y que se opone al desplazamiento entre ellos.

Una bola lanzada por el suelo de un salón se detiene; si la superficie es pulimentada tarda más en detenerse que si es rugosa, debido a la fuerza de rozamiento que le hace pasar de su estado de movimiento al estado de reposo.

Si se considera una superficie horizontal, suficientemente pulida de manera que el rozamiento sea despreciable en ella, la velocidad de la bola no debe ni aumentar ni disminuir. Galileo describe esta experiencia diciendo que el movimiento a lo largo de un plano horizontal sin rozamiento debe ser perpetuo, sin necesidad de que actúe sobre él fuerza alguna.

Las anteriores experiencias y razonamientos nos conducen a enunciar el principio llamado de inercia. Todo cuerpo permanece indefinidamente en su estado de reposo o de movimiento uniforme si sobre él no actúa ninguna fuerza exterior."

Texto 4 del [21] Análisis

Afirma que no es necesaria una fuerza para mantener un movimiento rectilíneo uniforme y atribuye esa frase a Galileo. Ya indicaremos en el apartado 7 que Galileo no se expresó así. Lo que propuso es que sin causas del tipo plano inclinado, que es algo externo al móvil, no habrá aceleración pero no dice que no exista algo tipo ímpetus, que es algo interno, para mantener la velocidad. No habla de fuerzas. Lo que se deduce de las experiencias del plano inclinado es a $\rightarrow \mathrm{f}$ con nuestra transposición didáctica [5] pero no, que $\mathrm{a}^{\prime} \rightarrow \mathrm{f}$ no sea cierta, ya que si se niega el antecedente, el consecuente puede ser verdadero o falso. Hay que aclarar explícitamente a qué llamamos fuerza, que es algo que en la descripción solo queda implícito. El calificativo exterior para las fuerzas, en el enunciado del principio de inercia da pie a hacer la distinción entre las fuerzas newtonianas y las de tipo impetus, pero no es suficiente con una insinuación. Es preciso, como hemos dicho anteriormente, una discusión explícita.

Texto 5 del [22]

"Principios fundamentales de la dinámica (p. 63)

Principio de la inercia

Enunciado 
Si sobre un cuerpo no actúa ninguna fuerza, permanecerá en reposo o en movimiento rectilíneo uniforme.

De otro modo: La materia es inerte. Un cuerpo por sí mismo no puede modificar su estado de reposo o movimiento. Por modificar su movimiento se entiende modificar su velocidad en módulo o en dirección.

Inercia en reposo...

Inercia en movimiento

La segunda parte resulta más difícil de admitir porque parece que contradice la experiencia ordinaria: $S i$ un cuerpo está en movimiento y no actúa sobre él ninguna fuerza, se mantendrá indefinidamente con movimiento rectilíneo y uniforme (velocidad constante).

Pero todo el mundo y quizá tú también piensa que para mantener en movimiento una vagoneta por una vía recta horizontal, con velocidad constante, hace falta empujarla, o sea, aplicar una fuerza y que si la fuerza deje de actuar, si te cansas de empujar, la vagoneta se parará.

Pero Galileo razonaba del siguiente modo: Cuando un cuerpo baja por un plano inclinado es fácil comprobar que su velocidad aumenta. Si en cambio sube, su velocidad va disminuyendo. Qué pasará si el plano es horizontal? La velocidad no aumentará ni disminuirá, se mantendrá constante.

Lanza una bola por un plano horizontal: recorre un cierto espacio y se detiene. Parece que la bola no acepta el razonamiento de Galileo. Pero repite la experiencia, primero con un suelo muy rugoso de tierra, después cemento, madera sin trabajar, madera bien pulida y encerada. Observas que, con el mismo impulso, la bola va cada vez más lejos. Puedes pensar que la bola no se detiene por si misma sino que hay una fuerza que la frena que va siendo cada vez más pequeña cuanto más pulimentada está la superficie: la llamamos fuerza de rozamiento. Si ahora imaginas un plano idealmente pulimentado, sin el más mínimo rozamiento, la bola impulsada por tu brazo, ante el asombro de tus compañeros, se movería indefinidamente en línea recta y con la misma velocidad."

Texto 5 del [22] Análisis

Tiene una estructura similar a varios de los anteriores: parte de $\mathrm{f}^{\prime} \rightarrow \mathrm{a}^{\prime}$, lo que es equivalente a $\mathrm{a} \rightarrow \mathrm{f}$ y la contrasta con $\mathrm{a}^{\prime} \rightarrow \mathrm{f}$. Ya hemos indicado que ni ella ni su negación se deducen lógicamente, a partir de la primera ley de Newton y que esto no elimina la posibilidad de movimientos rectilíneos uniformes mantenidos por "fuerzas". Aunque al afirmar que si está en movimiento y no actúa sobre él ninguna fuerza, está eliminando implícitamente las "fuerzas" asociadas al movimiento, en clase, deberá trabajarse una definición explícita de fuerza.

Retomemos la última frase: "Si ahora imaginas un plano idealmente pulimentado, sin el más mínimo rozamiento, la bola impulsada por tu brazo, ante el asombro de tus compañeros, se movería indefinidamente en línea recta y con la misma velocidad.. ${ }^{\text {Es }}$ to no elimina la posibilidad de que algún alumno piense que lo que ocurre es que le hemos dado "una fuerza. a la bola y que por eso continúa moviéndose. Siempre se va a necesitar la definición newtoniana de fuerza y una explicación del tipo [15], como la que hemos dado en el apartado 4.

Texto 6 del [23]
"Dinámica (p. 55)

Primera ley de Newton o principio de inercia

Puede enunciarse diciendo:

Todo cuerpo persiste en su estado de reposo o de movimiento rectilíneo uniforme si no interviene una fuerza que lo modifique.

Si un cuerpo sigue un camino recto y su velocidad aumenta (se acelera) es que actúa una fuerza o bien la resultante no nula de un sistema de fuerzas.

Si el cuerpo pierde velocidad (se decelera) es que actúa una fuerza en sentido contrario o se opone al movimiento. Intuitivamente podemos considerar una variación de velocidad como criterio para deducir la existencia de una fuerza. Si la velocidad no varía, es decir, si está en reposo (velocidad nula) continúa en reposo, si está en movimiento rectilíneo uniforme (velocidad constante) continúa con tal movimiento, podemos considerar que no hay fuerzas aplicadas o que la resultante que pueda haber es nula. Esto es lo que ocurre cuando un automóvil va en línea recta con velocidad constante. La fuerza del motor, que lo aceleraría está contrarrestada por las fuerzas debidas a rozamientos y el coche continúa a velocidad constante. Si la fuerza del motor supera a la de los rozamientos, el coche se acelerara y su velocidad aumenta. En caso contrario se decelera y podría llegar a pararse. Caso de no existir el rozamiento podríamos observar un movimiento uniforme persistente sin fuerzas aplicadas.

Estos hechos citados hacen que se pueda considerar que los cuerpos materiales están sometidos a la inercia, una especie de hábito (como se entiende la inercia en la vida corriente) que hace que los cuerpos persistan en su movimiento uniforme o que permanezcan en reposo si no hay ninguna causa externa que modifique su estado de movimiento o de reposo. Por eso al primer principio se le llama principio de inercia."

Texto 6 del [23] Análisis

Parte de la primera ley de Newton: $\mathrm{a} \rightarrow \mathrm{f}$ e introduce la proposición a' $\rightarrow \mathrm{f}$ 'que es equivalente a $\mathrm{f} \rightarrow \mathrm{a}$, que es la definición de fuerza newtoniana. Aquí sí que se introduce la definición de fuerza y no se pretende que se deduce de la primera ley de Newton. Conviene completarlo con una justificación del tipo [3] y [15] y una alusión a la segunda ley de Newton que es la que, cuantitativamente da sentido a la definición de fuerza. Como en todos los demás textos, después de la primera ley, se presentará la segunda. Creemos que después de estudiar la segunda ley se debería volver sobre la primera para mejorar explícitamente el concepto de fuerza.

\section{Consecuencias didácticas}

Hemos indicado al principio dos grandes líneas de objetivos que se pretende conseguir en la enseñanza secundaria, la comprensión de determinados conceptos, leyes y teorías y un conocimiento de aspectos del método científico, de pautas de pensamiento y de concepciones epistemológicas ajustadas al conocimiento científico propiamente dicho. En estas grandes líneas, hay preconcepciones de los 
alumnos que representan un obstáculo en la enseñanza y que han sido profusamente estudiadas

Evidentemente, una comprensión completa de los efectos y características de las fuerzas se obtendrá mediante el estudio de las tres leyes del movimiento, con un considerable trabajo adicional de discusión de situaciones y problemas de varios tipos, pero, ya desde el principio, parece razonable actuar, definiendo, bien por iniciativa del profesor o con una discusión previa con los alumnos, qué vamos a entender por fuerza, como hizo Newton (1687) en sus Principia y hemos reproducido anteriormente, descartando de entrada las "fuerzas mantenedoras de la velocidad", mediante la utilización explícita de las ideas indicadas por Johsua y Dupin (1993) que hemos destacado en el apartado IV, básicamente equivalentes a la navaja de Ockam, y, utilizando la definición en los razonamientos que hagamos en el apartado en el que se trate la primera ley de Newton. Al no actuar así, se aborda el concepto de fuerza de un modo oscuro, incompleto, inconsistente. Parece que se desafía a los alumnos a adivinar qué entendemos por "fuerza" sin darles suficientes herramientas. La definición de fuerza tiene un carácter arbitrario, esto es, como toda definición, dependiente del libre albedrío del autor de la misma; otra cuestión es la de la utilidad especial, que tiene la definición que se ha elegido (esta definición, con la segunda ley de Newton, permite conocer las aceleraciones, dadas las fuerzas) y que no la tendría otra definición, pero es arbitraria y por tanto no es natural ni evidente y a partir de una serie de observaciones, todas las personas no extraen el mismo concepto de fuerza como es perfectamente conocido, al menos a partir de la famosa tesis de L. Viennot (1979). Un alumno que utiliza en sus explicaciones un concepto tipo impetus medieval de fuerza no tiene por qué suponer que solo admitimos un concepto de fuerza newtoniano, si enunciamos la primera ley de Newton sin haber definido qué es una fuerza newtoniana y sin haber justificado la no utilización de las "fuerzas" asociadas a la velocidad, en nuestra teoría. Únicamente después de definir qué entendemos por fuerza tiene sentido el enunciado de la primera ley de Newton, en la que se menciona el término y deberemos además utilizar explícitamente esta definición (sabemos también que eso es insuficiente para que puedan tener una idea correcta y completa, y que para ello deberemos esperar al final del proceso, pero al menos, no sembraremos el camino de inconsistencias lógicas y estaremos obrando de un modo racional). Si los alumnos consideran que además de los tirones o empujones, originados por agentes externos al cuerpo sobre el que actúan, existe algo como el ímpetus medieval que es un agente interno, necesario para mantener la velocidad no parece razonable aclarar la situación siguiendo un camino, mediante el cual es lógicamente imposible hacerlo.

Por otro lado tenemos los aspectos del método científico, las pautas de pensamiento y las diferencias entre el pensamiento cotidiano y el pensamiento científico. Un elemento muy importante, como hemos indicado en el apartado 2, es que el conocimiento científico es altamente coherente y está organizado lógicamente de forma explícita. Hemos visto en algunos de los ejemplos analizados que se usan argumentos pretendidamente lógicos de un modo incorrecto por tanto no permiten una buena organización del conocimiento en la que se ponga de manifiesto la estructura lógica y la coherencia de los conceptos, que no constituyen una colección de piezas sueltas.

Por lo que se refiere a la especificación de conceptos, en [4] se indica que en la vida cotidiana hay poca precisión en la relación conceptos-referentes mientras que en la ciencia deben ser precisamente definidos para no conducir a inconsistencias. En los ejemplos que hemos visto, a veces se ha definido la fuerza anteriormente pero esa definición no se usa de modo explícito en los razonamientos del apartado en el que se trata la primera ley de Newton $\mathrm{Al}$ hacerlo así se muestra la ciencia como una colección de elementos, sin importar la relación entre ellos. Muchos alumnos piensan que aprender ciencias es aprender hechos y fenómenos que los científicos han ido descubriendo a lo largo del tiempo [6]. Esta idea sobre el aprendizaje de la ciencia es consistente con la concepción de la ciencia como un simple conjunto de hechos o fórmulas. Si se define el concepto de fuerza pero no se utiliza la definición en los razonamientos posteriores en los que intervenga dicho concepto, estaremos reforzando esa concepción de la ciencia como una colección de hechos y fórmulas sin una relación lógica y sin una coherencia.

\section{Consideraciones psicológicas e históricas}

El manejo correcto del condicional (si...entonces...) presenta graves dificultades, como ha sido comprobado en múltiples ocasiones, [24, 25, 26, 27, 28, 29]. El problema que nos ocupa es una cuestión de razonamiento humano y además en un contexto realista, no abstracto ni artificial. Tiene interés en varios ámbitos: (i) por una parte es de interés para los psicólogos del razonamiento, que buscan teorías que expliquen este comportamiento humano; (ii) por otra parte, para los historiadores y filósofos de la ciencia, por su relación con el papel de los paradigmas, aristotélico, del impetus medieval y newtoniano de las fuerzas a lo largo del tiempo; (iii) por último, la parte fundamental de este trabajo está destinada principalmente para los profesores, especialistas en didáctica, etc., que conocen la dificultad que representa para el proceso de enseñanza-aprendizaje, las ideas previas de los alumnos, sobre las fuerzas y su relación con el movimiento, sus pautas de pensamiento y sus concepciones epistemológicas.

i) Actualmente coexisten tres teorías que tratan de dar cuenta del comportamiento de la mente humana al resolver problemas de razonamiento [26] : (I) las teorías de reglas formales, que consideran al razonamiento como producto de la manipulación formal de reglas abstractas, (II) las que proponen la existencia de reglas específicas en la mente humana, según las cuales el razonamiento sería diferente en situaciones con la misma estructura lógica pero con contenidos diferentes y por último (III) los modelos mentales semánticos que consideran que el razonamiento se basa en la manipulación semántica de 
significados, sin reglas formales. Podemos citar a Piaget, Braine y Rips como representantes del grupo (I), a Minski, Rumelhart, Shank y Abelson, del grupo (II) y a Johnson-Laird y Byrne en el grupo (III). El interés para los psicólogos del razonamiento podría residir en responder a la pregunta ¿por qué algunos especialistas en ciencias han razonado incorrectamente con un condicional. Aunque la respuesta pueda ser que es un problema relacionado con el mundo real, que en la realidad hay una relación bicondicional y que en muchos de los casos, los errores de razonamiento consisten en esta utilización del condicional como si fuera bicondicional, no parece razonable aceptar, en un contexto académico y en una situación como ésta, con graves dificultades relacionadas con las ideas previas, una utilización bicondicional, aunque, tal vez en la vida cotidiana, en una situación similar, sea aceptable. Recordemos [4] que hay una serie de diferencias muy importantes entre el conocimiento científico y el conocimiento de la vida cotidiana en lo que respecta a las finalidades, los requerimientos, la comprensión, la validez, la especificación de los conceptos, la organización del conocimiento, los métodos, el control de calidad y la eficiencia. Aspectos análogos vemos [30] al hablar de las diferencias entre investigación e investigación científica. Si estamos introduciendo la Mecánica, y lo mismo deberíamos hacer con la enseñanza de cualquier otra parte de la física, hemos de aprovechar para introducir aspectos del comportamiento científico, marcando diferencias con la vida cotidiana.

ii) La historia de la ciencia puede ayudarnos a comprender la mezcla de paradigmas que aquí se produce [31]. Galileo, [32] como señala Heilbron [33] no se pronunció nunca sobre el concepto de fuerza como causa, que es el que nos interesa. Ha habido una evolución de las cuestiones relacionadas con la ley de inercia [34], comenzando con el paradigma aristotélico, el impetus medieval de Buridán, y continuando a través de Nicolás Copérnico, Giordano Bruno, Tycho Brahe, Johannes Kepler y Galileo Galilei. Especial interés tiene Galileo. Koyré [34] lo cita: "En realidad no comprendemos cuál es el principio y la virtud que mueve la piedra hacia abajo como tampoco sabemos qué es lo que la empuja hacia arriba cuando se separa de lo que la proyecta... de no ser, como he dicho, el nombre propio y especial de gravedad que hemos asignado al primero, mientras que para el otro empleamos el término más general de virtud impresa...". Aquí confluyen varias ideas: por un lado que el concepto de fuerza gravitatoria no está formado, se considera el peso como algo interno de los cuerpos; por otro lado, que considera la virtud impresa, también perteneciente al móvil, como la causa de que suba lo cual nos indica que consideraba necesaria una causa para este movimiento. Esto pone de manifiesto la dificultad del camino que ha conducido a la situación actual.

Tengamos en cuenta que esos paradigmas están mezclados en la mente de los alumnos, de una forma confusa y no podemos pretender aclararlos haciendo razonamientos poco explícitos o lógicamente incorrectos y que, encima potencian la confusión, al no aclarar de entrada qué en- tendemos por "fuerza" o si lo hemos hecho, al no utilizar explícitamente esa definición en los razonamientos.

\section{Líneas de investigación complementarias}

Contemplamos dos líneas de investigación que pueden ser consecuencia de ideas expuestas en este trabajo. Una de ellas estaría relacionada con la psicología del pensamiento y otra con la didáctica de la introducción a la Mecánica, que es la parte de la Física que hemos estado considerando.

Respecto a la psicología del pensamiento, podrían utilizarse estas cuestiones para estudiar las tres teorías que coexisten actualmente y que tratan de dar cuenta del comportamiento de la mente humana al resolver problemas de razonamiento, según indica el profesor Santamaría (1995) [28] y que hemos destacado en el apartado 6. Como curiosidad, y sin pretensión de que esto constituya una prueba científica, indicaremos que nuestra experiencia, al reflexionar sobre nuestro propio modo de reflexionar, nos lleva a decantarnos por los modelos mentales semánticos que consideran que el razonamiento se basa en la manipulación semántica de significados, sin reglas formales. Complementariamente se podrían utilizar los diferentes tipos de enunciados de la primera ley de Newton, que mostramos en la Tabla 2, con diferentes grupos de alumnos y comparar la comprensión de los mismos. Esto podría arrojar luz sobre la teoría de las reglas mentales y la de los modelos mentales como modos de explicar el razonamiento humano [35] También ayudaría a dilucidar cuál es el enunciado de la primera ley más aceptable didácticamente.

Por lo que respecta a la didáctica, sería interesante comparar, en esta parte de la enseñanza de la física, el método de enseñanza por descubrimiento [36, 37], con un método expositivo [38]. Nuestra experiencia nos hace decantarnos, en este caso, por un método expositivo y además, con una definición de fuerza propuesta por el profesor desde el principio, sin embargo, dejamos abierta la posibilidad de investigaciones experimentales, sistemáticas y rigurosas.

\section{Conclusiones}

1. Un enunciado de la primera ley del movimiento de Newton, equivalente al tradicional, dice que si un cuerpo está acelerado, actúa una fuerza neta sobre él. La proposición según la cual es necesaria una fuerza para mantener un movimiento rectilíneo uniforme se puede enunciar diciendo que si no está acelerado, actúa una fuerza sobre él y por tanto no puede deducirse ni su verdad ni su falsedad, a partir de la citada ley. Esto sería lógicamente incorrecto. Si partimos de un condicional y negamos el antecedente, el consecuente puede ser verdadero o falso.

2. Muchos alumnos, cuando comienzan el estudio de estas leyes, tienen un concepto de fuerza de tipo 
impetus medieval (agentes internos que mantienen la velocidad). La primera ley de Newton, sin una definición newtoniana de fuerza es lógicamente compatible con la existencia de este otro tipo de fuerzas. Por otra parte, Newton en sus Principia, define desde el comienzo de su exposición qué es para él fuerza. Sería conveniente hacer como Newton y dejar esta definición sentada desde el principio, para evitar los posibles malentendidos a los que el no hacerlo pudiera conducir, y por coherencia lógica, dado que es racional definir un término antes de usarlo. Esto no está reñido con discusiones previas con los alumnos sobre lo que piensan de una posible definición, pero ha de llegar un momento en el que se indique explícitamente la definición que aceptamos, y posteriormente, dicha definición debe utilizarse cuando sea necesario. Además deberemos utilizar esta definición en los razonamientos que hagamos en el apartado correspondiente a la primera ley de Newton.

3. La primera ley de Newton no es un bicondicional. No parece razonable en el contexto académico aceptar la interpretación del condicional como bicondicional, aunque el recíproco de este condicional sea cierto también. La física no es una ciencia formal como las matemáticas pero en ella tampoco es aceptable la imprecisión, como la que se produce en los razonamientos de la vida cotidiana.

4. El justificar explícitamente por qué debemos rechazar las fuerzas asociadas al mantenimiento de la velocidad constante es una buena ocasión para poner de manifiesto las referencias epistemológicas [3] y [15], que hemos mencionado anteriormente

\section{Referencias}

[1] J. Hierrezuelo y A. Montero, La ciencia de los alumnos (Laia/MEC, Barcelona, 1988).

[2] J.M. Campanario y J. Otero, Enseñanza de las ciencias, 18, 155, (2000).

[3] S. Johsua y J.J. Dupin, Introduction à la didactique des sciences et des mathématiques. (Universitaires de France, Paris, 1993), p. 23.

[4] F. Reif y J.H. Larkin, Journal of Research in Science Teaching, 28, 733 (1991).

[5] Y. Chevalard, La transposition didactique du savoir savant au savoir enseigné (La Pensée Sauvage, Grenoble, 1991), $2^{\mathrm{a}}$ ed.

[6] D. Hammer, Cognition and Instruction,12, 151 (1994).

[7] L. Viennot, Le raisonnement spontané en dynamique élementaire (Hermann, Paris, 1979).

[8] D.M. Watts, European Journal of Science Education 5, 217 (1983).

[9] J. Carrascosa Alis y D. Gil Pérez, Los errores conceptuales en la enseñanza de la Física. Un estudio sobre su persistencia, disponible en https: //www.researchgate.net/publication/303340630_ Los_errores_conceptuales_en_la_ensenanza_de_la_ Fisica_I_Un_estudio_de_su_persistencia
[10] J. Buridan, en: A Source Book in Medieval Science, editado por E. Grant (Harvard University Press, Cambridge, 1974).

[11] W.V. Quine, Filosofía de la lógica (Alianza, Madrid, 1970), $1^{\mathrm{a}} \mathrm{ed}$

[12] D. Hilbert y W. Ackermann, Elementos de lógica teórica (Estructura Y Funcion, Madrid, 1968).

[13] J.L. Anderson, American Journal of Physics 58, 1192 (1990).

[14] I. Newton, Principios matemáticos de la filosofía natural y su sistema del mundo (Editora Nacional, Madrid, 1982).

[15] G. Reale y D. Antiseri, Historia del pensamiento filosófico y científico (Herder, Barcelona, (1988), v. 1, p. 538.

[16] https://www.youtube.com/watch?v=69pwUDIak00

[17] A. Carcavilla, Revista de Enseñanza de la Física 27, 77 (2015).

[18] M. L. Calatayud Aleixandre, La construcción de las ciencias físico-químicas (Nau libres, Valencia, 1990).

[19] A. Candel, J. Satoca, J.B. Soler y J.J. Tent, Física y Química: $2^{\circ}$ de Bachillerato (Anaya, Salamanca, 1988)

[20] J.J. Lozano y J.L. Vigatá, Física y Química: Spin, $2^{\circ}$ de BUP (Alhambra, Madrid, 1985).

[21] J.M. Esteban, F. Marín, J.L. Negro y J.M. Vicente, Hacia la Física y la Química (Alhambra, Madrid, 1976).

[22] A.L. Lasheras y M.P. Carretero, Física y Química $2^{\circ}$ BUP (Vicens Vives, Barcelona, 1976).

[23] M. Pujal y J. Bozal, Física y Química (Teide, Barcelona, 1976).

[24] M. Carretero y J.A. García Madruga, in: Lecturas de psicología del pensamiento (Alianza, Madrid, 1984).

[25] J.A. Delval y A. Riviere, Revista de Psicología General y Aplicada 30, 825 (1975).

[26] P.C. Wason, en: Lecturas de psicología del pensamiento (Alianza, Madrid, 1984).

[27] M.D.S. Braine y D.P. O’Brien, Psychological Review 98, 182 (1991).

[28] C. Santamaría, Introducción al razonamiento humano (Alianza, Madrid, 1995)

[29] P.N. Johnson-Laird y R.M.J. Byrne, Psychological Review, 109, 646 (2002).

[30] W.C. Kyle Jr., Journal of Research in Science Teaching 17, 123 (1980).

[31] T.S. Kuhn, La estructura de las revoluciones científicas, disponible en http://www.sidocfeminista.org/ images/books/04434/04434_00.pdf.

[32] G. Galilei, Diálogo sobre los dos máximos sistemas del mundo ptolemaico y copernicano (Alianza Editorial, Madrid, 1995).

[33] W.F. Bynum, E.J. Browne y R. Porter, en: Diccionario de Historia de la Ciencia. (Herder, Barcelona, 1986).

[34] A. Koyré, Estudios galileanos (Siglo veintiuno, Madrid, 1980)

[35] N. Carriedo, S. Moreno, F. Gutierrez y G. Madurga, en: Manual práctico de psicología del pensamiento (Ariel, Barcelona, 2001).

[36] J.S. Bruner, Harvard Educational Review, 31, 21 (1961).

[37] J.S. Bruner, Desarrollo cognitivo y educación: Selección de textos por Jesús Palacios (Morata, Madrid, 1988).

[38] D.P. Ausubel, Psicología educativa: un punto de vista cognoscitivo (Trillas, Ciudad de México, 1976). 